\title{
Cécilia Claeys-Mekdade, une sociologue face à l'interdisciplinarité : éloge du doute méthodologique
}

\section{Propos recueillis par Agnès Pivot}

\author{
Cécilia Claeys-Mekdade ${ }^{a}$, Agnès Pivot ${ }^{b}$ \\ a Sociologue, DESMID/UMR Espace, Université de la Méditerranée, Département de Sciences humaines, Faculté des sciences de \\ Luminy, case 901, 163 avenue de Luminy, 13288 Marseille cedex 9, France \\ b Sociologue, LADYSS/NSS, Université Paris X, bâtiment Max Weber, 200 avenue de la République, 92001 Nanterre cedex, France
}

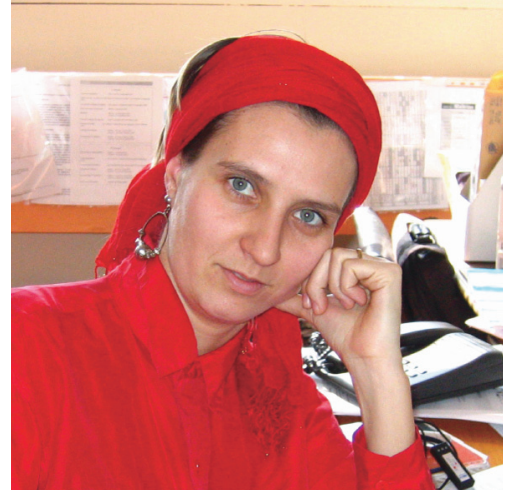

Agnès Pivot : Vous êtes une jeune sociologue au sein d'une équipe de recherches interdisciplinaire du CNRS, le Desmid, et vous enseignez dans une équipe pédagogique pluridisciplinaire de mathématiques et sciences sociales à la faculté des sciences de Luminy. Que pouvezvous nous dire, vous qui venez d'une discipline dite «molle» et qui travaillez avec une ou des disciplines dites «dures », des rapports entre ces disciplines et de votre expérience de l'interdisciplinarité ${ }^{1}$ ?

Auteur correspondant : C. Claeys-Mekdade,

Claeys.Mekdade@luminy.univ-mrs.fr

${ }^{1}$ Cet entretien a été engagé à la suite de l'école thématique Ladyss/NSS-Dialogues «La démarche interdisciplinaire dans le domaine de l'environnement : méthodes et outils pour partager les savoirs» (Aussois, 29 septembre-2 octobre 2003). Celle-ci avait pour objectif la mise en commun et en discussion des récentes expériences interdisciplinaires françaises, réalisées par des pionniers dans le domaine, comme par de jeunes chercheurs.
Cécilia Claeys-Mekdade : Avant de parler plus directement de mon expérience, je voudrais la situer dans son contexte institutionnel et scientifique. La transformation, au cours de l'histoire, du savant en scientifique a eu pour corollaire le découpage disciplinaire et un système d'évaluation par les pairs, dont l'universitaire contemporain a hérité et qu'il contribue peu ou prou à perpétuer. Au fur et à mesure que la connaissance s'étendait, se complexifiait, elle appelait une indispensable spécialisation des compétences, une nécessaire formalisation des protocoles d'expérimentation et d'investigation. L'institutionnalisation de ce découpage disciplinaire a créé ses propres jeux, enjeux et règles $\mathrm{du} \mathrm{jeu}^{2}$, mais aussi ses propres hiérarchies, souvent implicites, toujours présentes : sciences «dures» / sciences «molles», la taxinomie est incontestablement péjorative, j'aime pourtant à l'employer tant elle dit tout haut ce que de nombreux collègues pensent tout bas! On parle aussi de sciences exactes, sous-entendant alors, sans oser clairement le formuler, que les autres sciences seraient inexactes. Ne pourrait-on aussi parler, pourquoi pas, de sciences humaines et de sciences inhumaines? Ces hiérarchies fournissent une lecture tacite des grands découpages épistémologiques institutionnalisés (Sciences de l'homme et de la société, Sciences de la vie, Sciences mathématiques... ), ainsi que de la noblesse des spécialités au sein d'un domaine disciplinaire. Par exemple, au cœur des SHS, l'économétrie est représentée comme le côté dur des sciences molles, réciproquement,

\footnotetext{
2 Pour reprendre le jeu de mots cher à Pierre Bourdieu dans son analyse du champ scientifique (Bourdieu, 1997. Les Usages sociaux de la science : pour une sociologie clinique du champ scientifique, Paris, INRA).
} 
au sein des SDV, l'écologie tend à être considérée comme le côté mou des sciences dures.

Quels sont donc les ingrédients du mou et du dur? Le dur, et donc l'exactitude supposée, nécessite l'utilisation d'un catalyseur puissant : le chiffre et son maniement par les mathématiques. C'est une première condition nécessaire mais non pas suffisante, car, pour jouer aussi le rôle de durcisseur, ce catalyseur doit permettre d'échapper à toute source de mollesse de la science : les biais de l'observation dans toute leur diversité. Comment mieux échapper à ces inévitables biais de l'observation qu'en s'éloignant de l'observation elle-même? Ainsi, au sein du catalyseur mathématique, le chiffre devient lettre et l'observation s'incline devant l'abstraction. Telle est la noblesse de la recherche fondamentale!

Cette hiérarchie tacite est pourvoyeuse de complexes : complexe de supériorité pour les sciences dures et complexe d'infériorité pour les sciences molles. Au cours des années 1970, des volontés de développement interdisciplinaire ont été exprimées par quelques pionniers, las de leurs complexes respectifs : faisons donc des sciences molles des sciences plus dures en les enrichissant de mathématiques, suggèrent les uns! Faisons donc des sciences inhumaines des sciences plus humaines en les dotant d'un supplément d'âme, proposent les autres!

A.P. : Et donc plus concrètement, sur « le terrain », si je puis m'exprimer ainsi, dans les laboratoires et les programmes de recherches interdisciplinaires, cela donne quoi?

Cécilia Claeys-Mekdade : La mathématisation des SHS a produit son lot d'innovations et de formalisation. L'informatique a de surcroît permis de traiter de plus en plus vite des corpus de données de plus en plus grands. La mathématisation des SHS a aussi connu son lot d'excès, puis de désillusions. Les jeux et enjeux d'institutions ont fait le reste. Trente ans plus tard, qu'en reste-t-il? Pour le plus grand nombre des chercheurs en SHS, une performante boîte noire : logiciels d'analyse statistique des données quantitatives mais aussi qualitatives dont on ne connaît pas, ou mal, les choix mathématiques sousjacents. Les SHS sont-elles devenues plus dures qu'auparavant? Il ne semble pas, tant la hiérarchie dur/mou se révèle solidement ancrée dans les esprits. Que ce passet-il quand un sociologue rencontre un mathématicien? Les cas de figure sont pluriels, en voici quelques-uns vécus ou observés. Le trait commun à ces différentes situations est probablement le sentiment ambigu fait d'admiration, de défiance, de suspicion, voire de dénigrement réciproques. La première, la plus courante, est que l'on s'ignore, totalement, superbement. La seconde, évoquée à l'instant, est la rencontre occasionnelle qui cède ensuite la place au maniement de boites noires. La troisième situation observée est celle que l'on pourrait appeler la méthodologie forcenée. Dans ce cas, les échanges entre mathématiques et sociologie sont effectifs, réciproques et durables, mais l'outil tend à l'emporter, au point parfois de finir par déterminer la théorie. L'outil n'est dès lors plus un moyen, mais une fin en soi, n'offrant plus la possibilité de retour réflexif sur le sens de son utilisation dans une discipline ou une autre.

Ces différents rapports entre mathématiques et SHS eurent, et ont encore, des traductions didactiques et pédagogiques spécifiques, notamment dans le cadre de la filière MASS (mathématiques appliquées et sciences sociales) de la faculté des sciences de Luminy. La première, liée à l'ignorance réciproque, prit très logiquement la forme de cours qui s'ignoraient eux aussi mutuellement et ont été promulgués des enseignements de SHS d'un côté et de mathématiques de l'autre, mais surtout sans articulation. Les SHS, quant à elles, au mieux, enseignaient les rudiments du khi2, ce test de corrélation «de base», tandis que les mathématiques puisaient leurs exemples d'applications dans des domaines qui leur sont plus familiers, la physique et la biologie notamment. Ainsi, plusieurs générations d'étudiants (dont je faisais partie) ont acquis des compétences éparses sans avoir eu la possibilité de faire le lien entre les différentes disciplines qui leur étaient enseignées. Puis, avec la création d'un second cycle (une licence MASS et, plus récemment, une maîtrise, aujourd'hui devenue master), de réelles avancées didactiques et pédagogiques furent réalisées. La refonte du découpage des enseignements marque une amélioration significative. En effet, si un découpage disciplinaire perdure en partie, certains enseignements sont en revanche organisés sur le principe des différentes étapes de la démarche de recherche: 1 . Méthodologie d'enquête (produire et recueillir des données en SHS) ; 2 . Méthodes de traitement et d'analyse des données; 3 . Interprétation des données. Toutefois, ces enseignements réalisés par des sociologues et psychologues étaient menés parallèlement aux cours classiques de mathématiques et de statistiques.

A.P. : On retrouve ici l'importance de la formation des étudiants mais, au niveau de la recherche, selon vous, y a-t-il eu une avancée?

Cécilia Claeys-Mekdade : Parallèlement à ces efforts didactiques, un séminaire pluridisciplinaire mathématiques et SHS, initié et animé par des membres de l'équipe pédagogique MASS (un historien, Paul Allard, et une mathématicienne, Myriam Quatrini), fut lancé à l'attention des enseignants-chercheurs de la faculté des sciences de Luminy et de la région. Ce séminaire a montré la nécessité d'opérer un travail d'aller-retour régulier entre recherche et enseignements afin d'étayer et de consolider de possibles échanges interdisciplinaires. 
La nécessité heuristique de ces allers-retours a aussi pour corollaire des impératifs plus «bassement » stratégiques, liés aux modes actuels d'évaluation des carrières des enseignants-chercheurs. En effet, malgré son double statut, la carrière de l'enseignant-chercheur est essentiellement, sinon exclusivement, évaluée sur la base de ses productions scientifiques. De ce fait, l'investissement pédagogique constitue, en termes de carrière, au mieux une vocation gratuite, au pire un investissement à perte. Or, ce séminaire pluridisciplinaire a permis de voir naître de possibles vocations interdisciplinaires $\mathrm{du}$ point de vue des activités d'enseignement, mais aussi de recherche.

Au niveau de l'enseignement, la filière MASS, repensée dans le cadre de la réforme du système universitaire français, propose aujourd'hui plusieurs enseignements véritablement interdisciplinaires, animés par des binômes réunissant un enseignant de mathématiques et un enseignant de SHS. Au niveau de la recherche, un projet interdisciplinaire (réunissant deux historiens, une sociologue, un philosophe, une mathématicienne logicienne, une statisticienne et une informaticienne) portant sur l'analyse de données textuelles et l'émergence de nouveaux concepts est en cours de maturation. À suivre donc...

A.P. : Vous avez aussi travaillé avec des chercheurs des sciences de la vie. Que se passe-t-il quand un sociologue, vous en l'occurrence, rencontre un biologiste ou un écologue?

Cécilia Claeys-Mekdade : Là encore, admiration, défiance, suspicion, voire dénigrement réciproques sont au rendez-vous. Les premières collaborations entre SDV et SHS ont parfois pris des airs de chocs épistémologiques. Ici la «guerre des sciences » prend la forme d'une opposition de fond entre l'anthropocentrisme des SHS et le biocentrisme des SDV. L'incompréhension peut être grande, alors que les SDV tentent d'instrumentaliser les SHS en les utilisant comme outil de communication, les SHS répondent par un durcissement de leur posture critique, ne travaillant dès lors plus avec, mais sur les SDV (sinon contre elles), analysant les enjeux idéologiques, politiques et économiques portés par le discours de certains écologues (voir notamment l'analyse proposée par Jean-Louis Fabiani ${ }^{3}$ ). Ces frictions épistémologiques ne découragèrent toutefois pas les vaillants pionniers de l'interdisciplinarité.

Dès 1989, Nicole Mathieu et Marcel Jollivet appellent à une "réintroduction de la nature naturaliste dans les sciences sociales et de la nature sociale dans les sciences

\footnotetext{
${ }^{3}$ Fabiani J.-L., 1985. Science des écosystèmes et protection de la nature, in Cadoret, A. (Ed.), Protection de la nature : histoire et idéologie. De la nature à l'environnement, Paris, L'Harmattan, 75-93.
}

naturalistes ${ }^{4} »$. C'est dans cet esprit qu'est publié l'ouvrage collectif Sciences de la nature, sciences de la société : les passeurs de frontières ${ }^{5}$, puis créée la revue Natures Sciences Sociétés en 1993, qui souligne dès la première page de son premier numéro «la nécessité de l'interdisciplinarité ${ }^{6}$ », et annonce ensuite que "la recherche sur l'environnement sera interdisciplinaire ou ne sera pas $^{7} »$. Le ton de l'interdisciplinarité est donné.

Le PIREV du CNRS a aujourd'hui disparu, la nouvelle instance en cours de constitution, l'INSU. . . E, semble, à ce jour, encore hésitante dans sa façon d'appréhender les sciences sociales. Il n'y a toutefois pas lieu de s'inquiéter d'une disparition des demandes en SHS, car, de plus en plus, au niveau national et international, il est de bon ton dans les appels d'offres de valoriser les collaborations interdisciplinaires entre sciences dites «dures" et sciences dites "molles", notamment dans le cadre de l'environnement, entre SDV et SHS. Mais force est de constater que, dans ces appels d'offres, les sciences de l'homme font souvent office de "cerise sur le gâteau ". Les entrées et les problématiques sont produites par et pour les sciences de la vie qui sollicitent en second lieu les sciences de l'homme. De ce fait, les SHS et notamment la sociologie y sont appréhendées dans une logique directe d'aide à la décision et il est attendu d'elles qu'elles expliquent comment sensibiliser les populations aux problèmes d'environnement et comment changer leurs comportements ${ }^{8}$. Les SHS se retrouvent dès lors dans un rôle de discipline instrumentalisée. Qu'importe, pourrait-on être tenté de dire, si ceci permet in fine de faire avancer la science. Les SHS seraient-elles donc consentantes, devenant une "discipline sous influence», pour reprendre les mots de Marcel Jollivet?

A.P. : Durant l'école thématique d'Aussois, vous avez fait une remarque très intéressante sur la tendance des sciences sociales qui, en travaillant avec les sciences de la vie, peuvent passer d'un extrême à l'autre, d'une grande absence à une présence envahissante, par le biais du constructivisme. Cela peut décourager le biologiste qui se trouve, pour la première fois, face à une attitude de recherche qu'il estime trop critique. Qu'en est-il aujourd'hui, et diriez-vous tout de même

\footnotetext{
${ }^{4}$ Mathieu, N., Jollivet, M. (Eds), 1989. Du rural à l'environnement : la question de la nature aujourd'hui, Paris, ARF/L'Harmattan, 348.

${ }^{5}$ Jollivet, M. (Ed.), 1992. Sciences de la nature, sciences de la société : les passeurs de frontières, Paris, CNRS Éditions.

6 Jollivet, M., Legay, J.-M., Mégie, G., 1993. "Natures, sciences, sociétés » : un titre, un champ de réflexion, une ambition, Natures Sciences Sociétés, 1, 1, 1.

7 Jollivet, M., Pavé, A., 1993. L'environnement : un champ de recherche en formation, Natures Sciences Sociétés, 1, 1, 20.

${ }^{8}$ Henry, C., Jollivet, M., 2002. La question de l'environnement dans les sciences sociales en France. Éléments pour un bilan, Europaea, 1-2, anno VIII, 63-74.
} 
qu'il existe une plus-value engendrée par la pratique interdisciplinaire?

Cécilia Claeys-Mekdade : Oui, c'est vrai, en tentant de travailler avec les SDV, les SHS ont tour à tour travaillé pour, sur, voire contre elles. À ce titre, l'évolution de la sociologie de l'environnement française a l'aspect d'un mouvement de balancier, passant d'un anthropocentrisme tout critique des années 1970-1980 à un biocentrisme tout écologisant des années 1990 . Il s'agit peut-être de ses excès de jeunesse. Une voie intermédiaire permettant de rompre avec ce dualisme épistémologique caricatural paraît envisageable et souhaitable. Je m'explique : la pratique de l'interdisciplinarité permet de ne pas sombrer dans une critique absolue, en termes de constructivisme pur, des questions environnementales, par la confrontation de l'analyse sociologique avec l'analyse d'autres disciplines. En effet, la facilité pour le sociologue de ne voir dans l'environnement qu'une construction sociale, mêlant enjeu de pouvoir et rapports de domination, peut être évitée par l'apport des résultats des sciences de la vie et de l'univers. Les problèmes environnementaux sont ici pris en considération dans leur dimension scientifique et objective, rendant compte, à un moment donné, de l'état des connaissances scientifiques sur ces problèmes. En même temps, l'investissement fortement idéologique de certains écologues et biologistes par rapport aux problèmes environnementaux nous amène à maintenir une posture d'extériorité et d'objectivation de cette implication sociale du scientifique ${ }^{9}$.

Toutefois, comment tout à la fois travailler avec et sur les sciences de la vie? La pratique interdisciplinaire du doute méthodologique serait une voie possible. Pour ce faire, le sociologue devrait abandonner le monopole de la critique, de même que le biologiste devrait abandonner le monopole de l'exactitude scientifique. L'équité, le respect et la confiance mutuels sont dès lors indispensables. Et, en effet, comme les différentes interventions et les débats qui se sont déroulés lors de l'école thématique l'ont rappelé, l'interdisciplinarité ne se décrète pas, mais se construit sur la base d'échanges et d'affinités interpersonnels. C'est ce que nous essayons de faire dans la recherche interdisciplinaire (botanique/sociologie) en cours sur les processus d'invasion biologique ${ }^{10}$. Dans ce cas, il est demandé à la sociologie d'identifier les processus socioéconomiques responsables de la prolifération de

\footnotetext{
${ }^{9}$ Claeys-Mekdade, C., Jacqué, M., 2003. Is Sociology of the Environnement supposed to be "green"?, Ageing societies, new sociology, 6th Conference of the European Sociological Association, Murcia, 23-26 september.

10 Charpentier, A., Claeys-Mekdade, C., 2004. La biodiversitéà l'épreuve des Invasions Biologiques : perspective interdisciplinaire Biologie/Sociologie, Poster présenté lors des Deuxièmes Journées de l'Institut français de la biodiversité, Marseille, 25-28 mai.
}

deux espèces végétales (Baccharis et Cortaderia selloana). Tout en m'attelant à cette tâche, je mène conjointement avec la botaniste une réflexion critique sur le concept d'invasion biologique. Il s'agit dès lors de saisir la part idéologique d'un tel concept (le mot «idéologie» ne semble plus effrayer certains de nos collègues des sciences de la vie), sans ignorer pour autant la matérialité des processus biologiques, observés sur le terrain. Parallèlement à cette recherche, un séminaire "Invasions biologiques et sciences sociales » vient d'être lancé à l'initiative de plusieurs sociologues travaillant sur cette problématique, dans le cadre du programme de recherche "Invasion biologiques » du MEDD. Cette expérience naissante montre la double nécessité de collaborations interdisciplinaires et de retours réflexifs disciplinaires.

A.P. : J'ai l'impression, et vous m'en voyez fort heureuse, que votre conclusion sera donc plus optimiste que votre introduction?

Cécilia Claeys-Mekdade : Oui et j'en suis heureuse moi aussi, il y a tout un travail amorcé par les pionniers et continué par de jeunes chercheurs qui commence à porter ses fruits. Ainsi, le parti pris retenu par nos jeunes équipes est celui d'aller vers l'autre tout en sachant garder sa place, ou plutôt ses compétences. Il ne s'agit pas pour le sociologue de devenir mathématicien ou biologiste, et réciproquement. Si l'interdisciplinarité proposée bouscule la rigidité des frontières disciplinaires, elle ne conteste cependant pas la pertinence des découpages disciplinaires en tant que domaines de compétences. Comprendre l'autre, le questionner, sans le suspecter, ni se substituer à lui, ni établir de hiérarchie épistémologique entre les disciplines : tel est le sens de cette pratique interdisciplinaire du doute méthodologique que je propose. Un proche avenir nous dira si notre nouvelle génération de "passeurs de frontières » saura retenir les acquis de ses prédécesseurs tout en déjouant les pièges et les impasses de l'interdisciplinarité de demain!

Mais, pour que cet optimisme ne soit pas déçu, il me semble qu'un travail institutionnel de fond reste à faire en faveur du développement de l'interdisciplinarité. J'évoquais tout à l'heure le caractère timoré des premiers temps de la filière MASS, caractérisée par une juxtaposition d'enseignements disciplinaires. Il s'agissait aussi peut-être d'une forme de réalisme et de prudence. Aujourd'hui, dans l'équipe pédagogique MASS, nous sommes trois maîtres de conférences à être passées dans notre parcours d'étudiant par un Deug MASS, l'une est devenue mathématicienne, une seconde informaticienne, et moi... sociologue. On peut donc en conclure que la juxtaposition d'enseignements disciplinaires a aussi du bon et a fait ses preuves. En progressant vers une véritable interdisciplinarité, d'autres questions émergent : les nouvelles promotions 
d'étudiants sont véritablement interdisciplinaires, mais, pour continuer vers un troisième cycle (thèse), ils sont obligés de se plier au découpage ultradisciplinaire des écoles doctorales, d'abord, puis du CNU et des commissions de spécialistes qui règnent sur les procédures de recrutement. Pour risquer une analogie hardie, rappelons que la Révolution française fut un « procédé violent et rapide à l'aide duquel on a adapté
l'État politique à l'État social, les faits aux idées et les lois aux mœurs" (Tocqueville, 1986) ${ }^{11}$. La révolution interdisciplinaire reste-t-elle à faire? Ou, pour mobiliser un vocabulaire moins politique et plus épistémologique, le «paradigme» de l'interdisciplinarité doitil, pour sortir de sa marginalité, tordre le cou à la « science normale ${ }^{12}$, farouche gardienne du découpage disciplinaire?

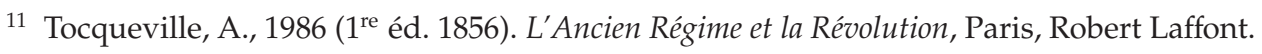

12 Kuhn, T.S., 1972 (éd. orig. 1962). La Structure des révolutions scientifiques, Paris, Flammarion. 